\title{
Resistencia a Cefepime en Aislamientos de Enterobacter cloacae provenientes de hospitales de Bogotá, Colombia
}

Elsa B. González-Mejía ${ }^{1}$, Emilia M. Valenzuela², José R. Mantilla-Anaya ${ }^{3}$, Aura L. LealCastro $^{4}$, Carlos H. Saavedra-Trujillo ${ }^{5}$, Javier Eslava-Schmalbach ${ }^{6}$ y Pedro SierraRodríguez $^{7}$

${ }^{1}$ Química Farmacéutica. M. Sc Instituto de Biotecnología. Universidad Nacional de Colombia. E-mail: ebgonzalezm@unal.edu.co

2 Química Farmacéutica. M. Sc. Instituto de Biotecnología. Universidad Nacional de Colombia. Teléfono 3165000 ext 16965.

${ }^{3}$ Químico Farmacéutico. M. Sc. Instituto de Biotecnología. Universidad Nacional de Colombia. E-mail: jrmantillaa@unal.edu.co

4 Médico. M. Sc. Departamento de Microbiología, Facultad de Medicina, Universidad Nacional de Colombia.E-mail: allealc@unal.edu.co

5 Médico Especialista en Medicina Interna e Infectología. Facultad de Medicina - Universidad Nacional de Colombia. E-mail: chsaavedrat@unal.edu.co

6 Médico. M. Sc. Ph. D (candidato) Facultad de Medicina, Universidad Nacional de Colombia. E-mail: jheslavas@unal.edu.co

7 Médico. M. Sc. Facultad de Medicina, Universidad Nacional de Colombia. E. Mail: pesiro@hotmail.com

Recibido 12 Diciembre 2005/Enviado para Modificación 15 Abril 2006/Aceptado 8 Junio 2006

\section{RESUMEN}

Objetivo Detectar la presencia de genes codificantes de beta-lactamasas que pueden conferir resistencia al cefepime en aislamientos de Enterobacter cloacae, para los cuales este antibiótico se considera una opción terapéutica importante.

Materiales y métodos Se analizaron 28 aislamientos provenientes de 4 hospitales de Bogotá, recolectados durante el año 2003. Se determinó fenotípicamente la producción de enzimas tipo cefalosporinasa y beta-lactamasas de espectro extendido. La presencia de genes bla codificantes para beta-lactamasas se detectó mediante amplificación por reacción en cadena de la polimerasa. Se evaluó por conjugación la posible transferencia de los genes bla que codifican para cefotaximasas.

Resultados Las pruebas microbiológicas mostraron que un $57 \%$ de los aislamientos eran productores de beta-lactamasas de espectro extendido. En $82 \%$ de los aislamientos se detectaron, genes bla $a_{\mathrm{TEM}}, b / a_{\mathrm{SHV}}$ y bla $a_{\mathrm{CTX}-\mathrm{M}}$. Siete de los 9 aislamientos que portaban genes bla CTX-M $_{\text {del }}$ grupo 1 estuvieron en el rango de intermedios o resistentes a cefepime. Estos aislamientos produjeron transconjugantes resistentes a cefotaxima.

Conclusión Se encontró relación entre la resistencia a cefepime y la presencia de cefotaximasas en E. cloacae. Los genes bla стх-м estuvieron pre- 
sentes en $32 \%$ de los aislamientos, indicando una diseminación importante en estos hospitales. La facilidad de transferencia de estos genes entre especies y géneros de enterobacterias es una razón importante para detectarlos y controlar su proliferación en el medio hospitalario. Estos resultados sugieren proceder con cautela en el uso de cefepime como alternativa terapéutica en las infecciones causadas por $E$. cloacae ante la posible presencia de cefotaximasas en estos aislamientos.

Palabras Clave: Enterobacter cloacae, resistencia bacteriana a las drogas, beta-Lactamasas, genes bacterianos (fuente: DeCS, BIREME).

\section{ABSTRACT \\ Resistance to Cefepime in Enterobacter cloacae isolates from hospi- tals in Bogotá, Colombia}

Objective The aim of this research was to detect the presence of genes encoding beta-lactamases which can confer cefepime resistance on Enterobacter cloacae isolates, meaning that this antibiotic may be considered an important therapeutic alternative.

Materials and methods $28 \mathrm{E}$. cloacae isolates collected from 4 hospitals in Bogotá during 2003 were analysed. Extended spectrum beta-lactamases and cephalosporinase production were phenotypically determined. The presence of extended spectrum beta-lactamase genes was determined by PCR amplification. Mating assays were done to determine the possible transfer of bla-genes encoding cefotaximases.

Results Microbiological tests detected $57 \%$ of extended-spectrum beta-lactamase-producing isolates. bla $a_{\mathrm{TEM}}$, bla $\mathrm{S}_{\mathrm{SHV}}$ and bla $\mathrm{C}_{\mathrm{CTX} \mathrm{M}}$ genes were detected by PCR in $82 \%$ of the isolates. 7 out of 9 isolates carrying group 1 bla genes were in the resistant or intermediate range for cefepime. These isolates produced cefotaxime-resistant transconjugants.

Conclusion A relationship was found between resistance to cefepime and

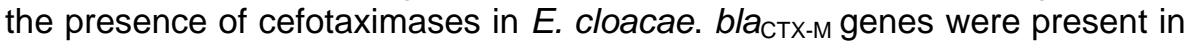
$32 \%$ of the isolates, indicating an significant spread in the hospitals being studied. The facility of these genes' transfer between other species and Enterobacteria genera becomes an important reason for detecting them and controlling their spread in hospital settings. These results suggest a cautious use of cefepime for treating infection caused by $E$. cloacae strains which might produce CTX-M enzymes.

Key Words: Enterobacter cloacae, drug resistance, beta-lactamase, bacterial gene (source: $\mathrm{MeSH}, \mathrm{NLM}$ )

a resistencia bacteriana a los antimicrobianos es un fenómeno inquietante que reduce las posibilidades de tratamiento de las enfermedades infecciosas y conduce a que enfermedades antes curables se 
vuelvan difíciles de tratar, mientras que otras que se creían controladas se hayan incrementado.

El Grupo para el Control de la Resistencia Bacteriana en Bogotá (GREBO), ha informado porcentajes de resistencia a cefalosporinas de tercera generación en E. cloacae entre 30 y $50 \%$ en unidades de cuidados intensivos (UCI) y no UCI en hospitales de Bogotá, durante los años 2001 al 2005 (1).

El Grupo de Epidemiología Molecular de la Universidad Nacional de Colombia, desarrolló durante el año 2003, el estudio "Epidemiología molecular aplicada a la identificación del modelo de la infección nosocomial causada por enterobacterias productoras de beta lactamasas de espectro extendido en tres hospitales de Bogotá". En esta investigación se planteó la aplicación de técnicas de biología molecular, en combinación con análisis epidemiológicos y clínicos para lograr un mayor conocimiento de las características genéticas de la población de enterobacterias productoras de betalactamasas de espectro extendido (BLEE), que circulan en algunos centros hospitalarios de Bogotá. Entre los resultados de este proyecto se encontró una alta proporción de cepas de enterobacterias productoras de BLEE y entre ellas una importante prevalencia de cefotaximasas (CTX-M) (2).

El género Enterobacter tiene resistencia natural a antibióticos betalactámicos como la ampicilina, amoxicilina y las cefalosporinas de primera generación, como resultado de la expresión de la beta-lactamasa cromosomal inducible AmpC. La hiperexpresión de esta enzima también confiere resistencia a las cefalosporinas de tercera generación (3).

Estudios recientes informan un aumento en la producción de BLEE en Enterobacter sp. lo cual también contribuye a la resistencia del microorganismo frente a las cefalosporinas de tercera generación (4). Es de anotar que las cefotaximasas confieren resistencia a cefepime y cefpirome (5).

Por otra parte, la detección de BLEE en E. cloacae suele verse afectada por la interferencia que genera la inducción de la enzima AmpC y por este motivo las BLEE pueden pasar desapercibidas. Este fenómeno causa inconvenientes desde la perspectiva de la elección de la terapia y también desde el punto de vista epidemiológico puesto que este microorganismo puede funcionar como reservorio de BLEE para otras especies $(6,7)$.

El cefepime se había considerado como posible terapia para el tratamiento de las infecciones causadas por este microorganismo, debido a que 
tiene una buena actividad in vitro, relacionada con la poca afinidad hacia la enzima AmpC. Sin embargo, el uso empírico de este antibiótico se ha limitado o suspendido en algunos centros hospitalarios debido al riesgo de falla terapéutica asociada con la presencia de BLEE en Enterobacter $(6,7)$.

\section{MATERIALES Y MÉTODOS}

Aislamientos bacterianos

Se estudió una colección de 28 aislamientos de E. cloacae de pacientes con infección intrahospitalaria obtenidos en un estudio previo (2).

La distribución de los aislamientos según el centro hospitalario del que provenían es la siguiente: 11 del Hospital Universitario Clínica San Rafael, 3 del Hospital de Santa Clara, 12 del Hospital de La Misericordia y 2 del Hospital El Tunal (Tabla 1).

Pruebas de identificación y susceptibilidad antimicrobiana La identificación y susceptibilidad a antimicrobianos fue realizada en cada centro hospitalario usando sistemas automatizados. En la Universidad Nacional de Colombia se ratificó la identificación mediante el sistema API20® de Biomerieux y se realizó inicialmente la prueba confirmatoria de BLEE mediante la técnica convencional de difusión en agar sugerida por el CLSIClinical and Laboratory Standards Institute (antes NCCLS-Nacional Comittee for Clinical Laboratory Standards) para K. pneumoniae y E. coli.

Determinación del fenotipo de producción de AmpC y BLEE

La determinación del fenotipo de producción de AmpC se realizó mediante la prueba de difusión con doble disco en agar Müeller - Hinton con discos de ceftazidima $(30 \mu \mathrm{g})$, cefoxitín $(30 \mu \mathrm{g})$ y cefotaxima $(30 \mu \mathrm{g})$, de manera que el disco de cefoxitín quedara en el centro de la caja y que la distancia entre éste y los discos de ceftazidima y cefotaxima fuera de $25 \mathrm{~mm}$ de centro a centro. El resultado de la prueba se consideró como positivo para AmpC inducible si el halo de inhibición para el disco de cefoxitín estaba en el rango de resistente, mientras que en los discos de ceftazidima o cefotaxima aparecía sensible y se confirmó si además se observaba un aplanamiento en el halo de inhibición de la ceftazidima o cefotaxima, en la proximidad del disco de cefoxitín. El resultado de la prueba se consideró positivo para AmpC desreprimida en los casos en los cuales se observó halo de inhibición en el rango de resistente para los tres antibióticos. Para la determinación del fenotipo de BLEE se utilizó la prueba de disco combinado, empleando discos de cefepime y cefepime combinado con ácido clavulánico, ubicando los discos a una distancia de $30 \mathrm{~mm}$ de centro a centro. El resultado se consideró posi- 
tivo para BLEE cuando se observó un incremento en el diámetro del halo de inhibición mayor o igual a $5 \mathrm{~mm}$.

Detección de genes bla mediante Reacción en Cadena de la Polimerasa (Polymerase Chain Reaction - PCR)

La detección por PCR de genes bla se realizó utilizando como molde el ADN presente en lisados bacterianos obtenidos mediante el procedimiento de lisis por ebullición.

Las secuencias de los iniciadores utilizados fueron:

bla $_{\text {TEM }}$ : TEMF - 5'-CTTATTCCCTTTTTTGCGGC-3' y TEMR- 5'GGTCTGACAGTTACCAATGC-3';

bla $a_{\mathrm{SHV}}$ : SHVF - 5'-TTATCTCCCTGTTAGCCACC-3' y SHVR - 5'GATTTGCTGATTTCGCTGG-3';

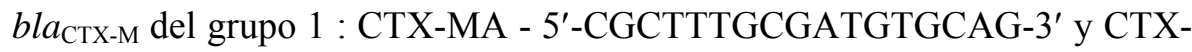
MB - 5'-ACCGCGATATCGTTGGT-3';

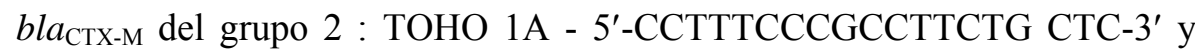
TOHO 1B - 5'-ACGCTACCCCTGCTATTT-3';

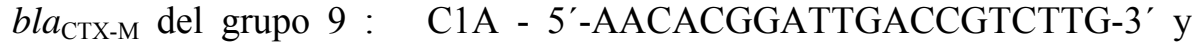
C1B - 5'-TTACAGCCCTTCGGCGAT-3'.

Los productos de las amplificaciones se evaluaron mediante electroforesis en geles de agarosa y los resultados se visualizaron y digitalizaron en el digitalizador Gel Doc ${ }^{\circledR}$ (Bio-Rad).

Conjugación

Los aislamientos portadores de genes $b a_{\text {CTX-M }}$ del grupo 1 fueron conjugados con E. coli J-53-2 y se obtuvieron transconjugantes que se seleccionaron en medio sólido con cefotaxima y rifampicina. En estos también se detectó el gen $b l a_{\text {СТХ-м }}$ por PCR con los iniciadores indicados anteriormente para el grupo 1.

\section{RESULTADOS}

Determinación del fenotipo de expresión de AmpC y BLEE

La distribución de los aislamientos de acuerdo con la expresión de AmpC fue de 17 desreprimidas $(60,7 \%), 9$ inducibles $(32,1 \%)$ y 2 presuntivas de BLEE $(7,1 \%)$. La determinación de BLEE por la prueba microbiológica con cefepime, dio un total de 17 aislamientos positivos (57\%) y 11 aislamientos negativos (43\%) (Tabla 1$)$.

Detección de genes codificantes de BLEE mediante PCR 
Se detectaron genes $b l a_{\mathrm{TEM}}$ en $21(75 \%)$ de los 28 aislamientos 12 aislamientos presentaron genes $b l a_{\mathrm{SHV}}$, nueve aislamientos presentaron genes

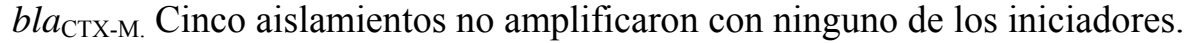

Tabla 1. Características genéticas, bioquímicas y microbiológicas de aislamientos de E. cloacae

\begin{tabular}{|c|c|c|c|c|c|c|}
\hline \multirow{2}{*}{ Aislamiento $^{\mathrm{a}}$} & \multirow{2}{*}{$\begin{array}{c}\text { Patrón de resistencia } \\
\text { Antibiótica }^{\mathrm{b}}\end{array}$} & \multirow{2}{*}{$\begin{array}{l}\text { Expresión de } \\
\text { AmpC }\end{array}$} & \multirow{2}{*}{$\begin{array}{l}\text { Detección } \\
\text { de BLEE }\end{array}$} & \multicolumn{3}{|c|}{$\begin{array}{c}\text { Genes bla } \\
\text { tipo }\end{array}$} \\
\hline & & & & TEM & SHV & $\begin{array}{c}\text { CTX- } \\
M\end{array}$ \\
\hline $1-014$ & AK, CRO, FOX, CTX, FEP & Inducible & BLEE & + & - & + \\
\hline $1-017$ & AK, CRO, FOX, CTX, CAZ, FEP & Desreprimida & BLEE & + & + & - \\
\hline $1-026$ & AK, CRO, FOX, CTX, CAZ, FEP & Desreprimida & BLEE & + & + & - \\
\hline $2-010$ & AK, CRO, FOX, CTX, CAZ, FEP & Desreprimida & BLEE & + & - & + \\
\hline $2-032$ & AK, CRO, FOX, CAZ & Inducible & BLEE & + & + & - \\
\hline $2-033$ & AK, CRO, FOX, CTX, CAZ, FEP & Desreprimida & BLEE & + & - & + \\
\hline $2-048$ & AK, CRO, FOX, CTX, CAZ & Desreprimida & BLEE & - & - & - \\
\hline $2-054$ & AK, CRO, FOX & Inducible & No BLEE & - & - & - \\
\hline $2-062$ & $\mathrm{AK}, \mathrm{CRO}, \mathrm{CAZ}$ & BLEE presuntiva & BLEE & - & + & - \\
\hline $2-080$ & AK, CRO, FOX, CTX, CAZ & Desreprimida & BLEE & + & + & - \\
\hline $2-128$ & CRO, FOX, CTX, FEP & Inducible & BLEE & + & - & + \\
\hline $2-135$ & AK, CRO, CTX, CAZ & BLEE presuntiva & BLEE & - & + & + \\
\hline $2-272$ & AK, CRO, FOX, CTX, FEP & Inducible & BLEE & + & - & + \\
\hline $2-274$ & AK, CRO, FOX, CTX, FEP & Inducible & BLEE & + & - & + \\
\hline $2-294$ & CRO, FOX, CTX, CAZ & Desreprimida & BLEE? & + & - & + \\
\hline $3-013$ & AK, CRO, FOX, CTX, CAZ & Desreprimida & BLEE? & + & + & - \\
\hline $3-107$ & CRO, FOX, CTX, CAZ & Desreprimida & No BLEE & - & - & - \\
\hline $4-545$ & AK, CRO, FOX, CTX, CAZ & Desreprimida & BLEA & + & - & - \\
\hline $4-788$ & AK, CRO, FOX, CAZ, FEP & Inducible & BLEE & + & + & - \\
\hline $4-827$ & AK, CRO, FOX, CAZ & Inducible & BLEE & + & + & - \\
\hline $4-1228$ & AK, CRO, FOX, CTX, CAZ & Desreprimida & BLEA & + & - & - \\
\hline $4-2273$ & AK, CRO, FOX, CTX, CAZ, FEP & Desreprimida & BLEA & + & - & - \\
\hline $4-3787$ & AK, CRO, FOX, CTX, CAZ & Desreprimida & BLEE? & + & + & - \\
\hline $4-3867$ & AK, CRO, FOX, CTX, CAZ & Desreprimida & BLEE? & + & + & - \\
\hline $4-5747$ & AK, CRO, FOX, CTX, CAZ & Desreprimida & BLEE & + & + & - \\
\hline $4-5935$ & AK, CRO, FOX, CTX, CAZ & Desreprimida & No BLEE & - & - & - \\
\hline $4-6914$ & FOX & Inducible & No BLEE & - & - & - \\
\hline $4-7649$ & AK, CRO, FOX, CTX, CAZ, FEP & Desreprimida & BLEE & + & - & + \\
\hline
\end{tabular}


Fue más frecuente encontrar varios genes codificantes de BLEE en un mismo aislamiento que encontrar uno solo. La combinación más observada fue la de $b l a_{\mathrm{TEM}} / b l a_{\mathrm{SHV}}$, seguida de $b l a_{\mathrm{TEM}} / b l a_{\mathrm{CTX}-\mathrm{M}}$. En general los genes más encontrados son los que codifican para enzimas tipo TEM (Tabla 1).

Conjugación

Todos los aislamientos portadores de genes bla $_{\text {СTX-M }}$ produjeron transconjugantes con E. coli J-53-2, en los cuales se detectaron mediante PCR portadores de genes $b l a_{\text {СТХ-M. }}$ Los transconjugantes adquirieron el fenotipo de BLEE y resistencia a cefotaxima. Un $44 \%$ de los transconjugantes adquirió resistencia a cefepime.

\section{DISCUSIÓN}

Se observó diferencia entre los resultados obtenidos mediante la prueba microbiológica de disco combinado para detectar la producción de BLEE y la amplificación por PCR. Con este procedimiento se detectaron genes bla en 23 de 28 aislamientos (82\%), mientras que con la prueba de disco combinado se confirmaron 17 aislamientos productores de BLEE (Tabla 1). De los once aislamientos que dieron resultado negativo en la prueba microbiológica, cuatro (2-054, 3-107, 4-5935, 4-6914) no amplificaron con ninguno de los iniciadores, por lo cual se les consideró como no BLEE (Tabla 1). Otros tres aislamientos (4-545, 4-1228 y 4-2273) solamente amplificaron con iniciadores para genes tipo TEM, resultado que permite suponer que se trata de genes que codifican para la beta-lactamasa TEM1 que no se considera BLEE sino BLEA (beta-lactamasa de espectro ampliado). Los cuatro aislamientos restantes amplificaron con alguno de los iniciadores pero no dieron positiva la prueba microbiológica para BLEE con cefepime, por lo cual se clasificaron como BLEE dudosos (BLEE? en la Tabla 1). La característica común de estos cuatro aislamientos es que tienen fenotipo de $\mathrm{AmpC}$ desreprimida.

Debido a la dificultad que existe para la interpretación de las pruebas microbiológicas por la falta de criterios precisos para este tipo de microorganismo, algunos autores opinan que es imposible implementar un método confiable de detección de BLEE con base en la susceptibilidad a las cefalosporinas, para organismos que producen $\mathrm{AmpC}$ (8). Por esta razón se sugiere determinar el fenotipo de AmpC en este microorganismo así como utilizar pruebas moleculares para complementar los resultados de la prueba microbiológica de detección de BLEE. 
En el presente trabajo, se encontró un $60,7 \%$ de aislamientos con expresión desreprimida de AmpC y 32,1 \% inducibles, lo cual puede indicar una fuerte presión selectiva por la terapia antibiótica.

Con base en estos resultados se puede considerar que el mecanismo de resistencia que predomina en los aislamientos es la hiperproducción de la enzima AmpC. Si se tratara del único mecanismo, el cefepime podría ser una alternativa para el tratamiento de estas infecciones (9). Sin embargo, es posible inferir una relación entre la presencia de genes codificantes para BLEE de tipo CTX-M-1 y la resistencia a cefepime, pues se encontró que de los 9 aislamientos que amplificaron con estos iniciadores, 7 presentaron fenotipo de resistencia al cefepime (interpretación del halo de inhibición en el rango de resistente o intermedio). En esas condiciones es aún más importante contar con resultados que permitan determinar con claridad la presencia de BLEE en estos aislamientos.

Las pruebas automatizadas y las pruebas microbiológicas convencionales para la detección de BLEE no fueron precisas para determinar la presencia simultánea de estas enzimas en los aislamientos. Esta confirmación sólo fue posible mediante la aplicación de la PCR, con la cual se pudo detectar un alto porcentaje de BLEE del tipo CTX-M. En efecto, un estudio realizado durante el año 2005, reveló una importante presencia de diferentes tipos de beta-lactamasas de los grupos CTX-M-1 y CTX-M-2 en aislamientos de origen hospitalario y también en infecciones adquiridas en la comunidad (10).

A pesar de su importancia, en el medio hospitalario no se encuentran disponibles estas alternativas de diagnóstico. La detección oportuna de este tipo de enzimas permite guiar la decisión del médico en la elección del antibiótico más adecuado. En tal caso, un carbapenémico es la única alternativa disponible; en segundo lugar, permite prevenir la diseminación de esta resistencia que puede pasar inadvertida y convertirse en un fenómeno difícil de contener en el medio hospitalario $(6,7)$.

Agradecimientos. Los autores expresan su agradecimiento a la Universidad Nacional de Colombia y al Instituto Colombiano para el Desarrollo de la Ciencia y la Tecnología-COLCIENCIAS por proveer la financiación y el soporte tecnológico necesarios para la realización de este trabajo.

Conflictos de interés: Ninguno

\section{REFERENCIAS}


1. GREBO - Grupo de Estudio de la Resistencia en Bogotá. Perfiles de Resistencia 2001-2005 [Internet]. Disponible en http://www.grebo.org/perfiles.asp. Consultado en mayo de 2006.

2. Mantilla JR, Valenzuela EM, González EB, Méndez AM, Leal AL, Sierra P, et al. Alta prevalencia de cefotaximasas del grupo CTX-M-1 en Enterobacteriaceae asociadas a infección intrahospitalaria en Bogotá. Infectio 2004; $8: 143$.

3. Livermore D. $\beta$-Lactamase-mediated resistance and opportunities for its control. J Antimicrob Chemother 1998; 41 (Suppl. D): 25-41.

4. Pai H, Hong JY, Byeon JH, Kim YK, Lee HJ. High prevalence of extended spectrum $\beta$-lactamase-producing strains among blood isolates of Enterobacter spp. collected in a tertiary hospital during an 8-year period and their antimicrobial susceptibility patterns. Antimicrob Agents Chemother 2004; 48:3159-61.

5. Bonnet R. Growing group of extended-spectrum $\beta$-lactamases: the CTX-M enzymes. Antimicrob Agents Chemother 2004; 48:1-14.

6. Gottlieb T, Wolfson C. Comparison of the MICs of cefepime for extended-spectrum $\beta$-lactamase-producing and non-extended spectrum $\beta$-lactamase-producing strains of Enterobacter cloacae. J Antimicrob Chemother 2000; 46:330-1.

7. Naumiuk L, Samet A, Dziemaszkiewicz E. Cefepime in vitro activity against derepressed extended-spectrum $\beta$-lactamase (ESBL)-producing and nonESBL-producing Enterobacter cloacae by a disc diffusion method. J Antimicrob Chemother 2001; 48: 321-2.

8. Schwaber MJ, Raney PM, Rasheed JK, Biddle JW, Williams P, McGowan JE, et $a l$. Utility of NCCLS guidelines for identifying extended-spectrum $\beta$-lactamases in non-Escherichia coli and non-Klebsiella spp. of Enterobacteriaceae. J Clin Microbiol 2004; 42:294-8.

9. Crowley B, Ratcliffe G. Extended-spectrum $\beta$-lactamases in Enterobacter cloacae: underestimated but clinically significant! J Antimicrob Chemother 2003; 51:1316-7.

10. Valenzuela EM, Mantilla JR, Reguero MT, González EB , Pulido IY, Llerena ID, et al. Detection of CTX-M-1, CTX-M-15, and CTX-M-2 in clinical isolates of Enterobacteriaceae in Bogotá, Colombia. J Clin Microbiol 2006; 44:1919-20. 